International Journal of Distributed and Parallel Systems (IJDPS) Vol.3, No.2, March 2012

\title{
INTELLIGENT WIRELESS COMMUNICATION SYSTEM USING COGNITIVE RADIO
}

\author{
Asma Amraoui ${ }^{1}$, Badr Benmammar ${ }^{1}$, Francine Krief $^{2}$, Fethi Tarik Bendimerad ${ }^{1}$ \\ ${ }^{1}$ LTT Laboratory of Telecommunication Tlemcen, UABT, Algeria \\ ${ }^{2}$ LaBRI Laboratory Bordeaux 1 University 33400 Talence, France \\ \{amraoui.asma, badr.benmammar, ftbendimerad\}@gmail.com, krief@labri.fr
}

\begin{abstract}
The increasing demand for wireless communication introduces efficient spectrum utilization challenge. To address this challenge, cognitive radio $(C R)$ is emerged as the key technology; which enables opportunistic access to the spectrum. CR is a form of wireless communication in which a transceiver can intelligently detect which communication channels are in use and which are not, and instantly move into vacant channels while avoiding occupied ones. This optimizes the use of available radio-frequency $(R F)$ spectrum while minimizing interference to other users. In this paper, we present a state of the art on the use of Multi Agent Systems (MAS) for spectrum access using cooperation and competition to solve the problem of spectrum allocation and ensure better management. Then we propose a new approach which uses the CR for improving wireless communication for a single cognitive radio mobile terminal (CRMT).
\end{abstract}

\section{KEYWORDS}

Cognitive Radio, wireless communications, mobility, Artificial Intelligence, Multi Agent Systems.

\section{INTRODUCTION}

The Cognitive Radio (CR) was presented officially by Joseph Mitola in 1999, and since, this concept has been very popular with researchers in several fields such as telecommunications, artificial intelligence, and even philosophy. Joseph Mitola has defined the CR as "a radio that employs model-based reasoning to achieve a specified level of competence in radio-related domains" [1].

Most researches on CR networks have focused on the exploitation of unused spectrum. However, the CR nodes possess the necessary qualities to make a considerable progress in the reliability of wireless networks [2], which has been less explored, so that is why we were interested by improving the wireless link reliability of a video conferencing application.

The aim of our paper is to propose a technique to improve wireless communication to a video conferencing application for a mobile terminal using the CR for only one CRMT. For this, it seemed appropriate to choose a scenario on which we will apply our approach. Our technique is based on machine learning. In our future work, we will seek to improve real-time application performance related to many CRMT based on MAS.

In this paper, we first present how Artificial Intelligence techniques can be used in the Cognitive Radio networks. Then, we present a state of the art concerning the use of MAS in the context of CR, after that we explain our approach and describing the proposed scenarios and finally, the results of our experimentation. 
International Journal of Distributed and Parallel Systems (IJDPS) Vol.3, No.2, March 2012

\section{ARTIFICIAL INTELLIGENCE AND COGNITIVE RADIO}

Artificial intelligence (AI) techniques for learning and decision making can be applied to design efficient cognitive radio systems. The concept of machine learning was applied to CR for capacity maximization and dynamic spectrum access. Different learning algorithms can be used in CR networks (Hidden Markov Model, neural networks, genetic algorithms, decision trees, fuzzy logic or classification algorithms) [3].

CRs need to have the ability to learn and adapt their wireless transmission according to the ambient radio environment. Intelligent algorithms such as those based on machine learning, genetic algorithms, and fuzzy control are therefore key to the implementation of CR technology. In general, these algorithms are used to observe the state of the wireless environment and build knowledge about the environment. This knowledge is used by a cognitive radio to adapt its decision on spectrum access. For example, a secondary user can observe the transmission activity of primary users on different channels. This enables the CR to build knowledge about the primary users' activity on each channel. This knowledge is then used by the CR to decide which channel to access so that the desired performance objectives can be achieved (e.g. throughput is maximized while the interference or collision caused to the primary users is maintained below the target level) [4].

\subsection{Neural Networks}

A multilayered neural network was used to model and estimate the performances of IEEE 802.11 networks. This neural network provides a black-box model for the non-linear relationship between the inputs and the outputs. This neural network model can learn from training data which can be obtained in an on-line manner when the real-time measurement data are available. Therefore, this model is suitable for a cognitive radio network for which a prompt response to the changing radio environment is required from an unlicensed user [4].

\subsection{Fuzzy Logic}

Fuzzy logic is often combined with neural networks that can adapt to the environment during the evolution of a CR system.

A fuzzy logic control system can be used to obtain the solution to a problem given imprecise, noisy, and incomplete input information.

In short, instead of using complicated mathematical formulations, fuzzy logic uses humanunderstandable fuzzy sets and inference rules to obtain the solution that satisfies the desired system objectives. The main advantage of fuzzy logic is its low complexity. Therefore, fuzzy logic is suitable for real-time cognitive radio applications in which the response time is critical to system performance.

In general, there are three major components in a fuzzy logic control system: fuzzifier, fuzzy logic processor, and defuzzifier. While the fuzzifier is used to map the crisp inputs into fuzzy sets, the fuzzy logic processor implements an inference engine to obtain the solution based on predefined sets of rules. Then, the defuzzifier is applied to transform the solution to the crisp output [4].

\section{Multi Agent Systems And Cognitive Radio}

The association of MAS and the CR can provide a great future for the optimal management of frequencies (in comparison with the rigid control techniques proposed by the telecommunications operators). In the case of use of unlicensed bands, the CR terminals have to coordinate and cooperate to best use the spectrum without causing interference. 
International Journal of Distributed and Parallel Systems (IJDPS) Vol.3, No.2, March 2012

In [25], the authors propose an architecture based on agents where each CR terminal is equipped with an intelligent agent, there are modules to collect information about the radio environment and of course the information collected will be stored in a shared knowledge base that will be accessed by all agents. The proposed approach is based on cooperative MAS (the agents have common interests). They work by sharing their knowledge to increase their collective and individual gain.

Agents are deployed on the PUs and SUs terminals and cooperate with each other in the works proposed in [23] [16] [17]. By cooperative MAS, we mean that PU agents exchanged t-uples of messages in order to improve themselves and the neighbourhood of SU agents. They propose that the SUs should make their decision based on the amount of available spectrum when they find a suitable offer (without waiting for response from all PUs). In other words, the SU agent should send messages to the appropriate neighbour PU agent and of course the concerned PU must respond to these agents to an agreement on sharing the spectrum. After the end of the spectrum use, the SU must pay the PU.

A comparison is made in [16] between an agent and a CR. Basically, both of them are aware of their surrounding environments through interactions, sensing, monitoring and they have autonomy and control over their actions and states. They can solve the assigned tasks independently based on their individual capabilities or can work with their neighbours by having frequent information exchanges.

To make the CR systems practical, it requires that several CR networks coexist with each other. However, this can cause interference. The authors of [14] think that to remedy this problem, the SU can cooperate to sense the spectrum as well as to share it without causing interference to the PU. For this, they propose schemes to protect the PU from interferences by controlling the transmission power of the cognitive terminal.

In [20] [15], the authors propose cooperation between PUs and SUs and between SUs only. Agents are deployed on the user's terminals to cooperate and result in contracts governing spectrum allocation. SU agents coexist and cooperate with the PU agents in an Ad hoc CR environment using messages and mechanisms for decision making. Since the internal behaviours of agents are cooperative and selfless, it enables them to maximize the utility function of other agents without adding costs result in terms of exchanged messages.

However, the allocation of resources is an important issue in CR systems. It can be done by making the negotiation among SUs [22] [24]. In [22] the authors propose a model based on agents for the spectrum trading in a CR network. But instead of negotiating spectrum directly with the PU and SU, a broker agent is included. This means that the equipment of PU or SU does not require much intelligence as it does not need to perform the spectrum sensing. The objective of this trading is to maximize the benefits and profits of agents to satisfy the SU. The authors proposed two situations, the first uses a single agent who will exploit and dominate the network, in either case there will be several competing agents.

The authors in [13] study the use of CR in wireless LANs and the possibility of introducing the technology of agents, in other words they try to solve the problem of radio resources allocation by combining resources management WLAN in a decentralized environment, this by using MAS. For this, they propose an approach based on MAS for sharing information and decisions distribution among multiple WLANs in a distributed manner.

Interference from the acquisition of the channels in a cellular system during Handovers can be reduced according to [11] [7] using a CR to manage the handover. Indeed, the mobility of the device imposes a different behaviour when changing zones. The terminal must ensure 
International Journal of Distributed and Parallel Systems (IJDPS) Vol.3, No.2, March 2012

service continuity of applications and the effective spectrum management. The authors propose an approach that uses negotiation, learning, reasoning and prediction to know the needs of new services in modern wireless networks. They propose an algorithm to be executed by the mobile terminal during the cognitive phase of handover.

The MAS contains several intelligent agents interact with each other. Each agent can sense and learn. The agent can select behaviours based on local information and attempt to maximize overall system performance. In [8], they described a new approach based on multiagent reinforcement learning which is used in CR networks with ad hoc decentralized control. In other words, they set up several CR scenarios and affect each case a reward or penalty. The results of this approach have shown that with this method, the network can converge to a fair spectrum sharing and of course it reduces interferences with PUs.

A very interesting approach is proposed in [18] where the authors have applied reinforcement learning RL on single-agent (SARL) and Multi-Agent (MARL) to achieve the sensitivity and the intelligence. They show in their results that the SARL and MARL perform a joint action that gives better performance across the network.

They finally said reinforcement learning algorithm is adapted too be applied in most application schemas.

In the solution proposed in [10], a learning mechanism as the local MARL is available for each agent. The Local Learning provides a reward for each agent so that it can make the right decision and choose the best action. They modeled each SU node as a learning agent because the transmitter and receiver share a common result of learning or knowledge. The authors presented the LCPP (Locally Confined Payoff Propagation) which is an important function of reinforcement learning in MAS to achieve optimality in the cooperation between agents in a distributed CR network.

A channel selection scheme without negotiation is considered for multi-user and multichannel in [24]. To avoid collision incurred by non-coordination, each SU learns to select channels based in their experiences. The MARL is applied in the context of Q-learning by considering the SUs as part of environment. In such a scheme, each SU senses channels and then selects a slowed frequency channel to transmit the data, as if no other SU exists. If two SUs choose the same channel for data transmission, they will collide with each other and the data packets cannot be decoded by the receiver. However, the SUs can try to learn how to avoid each other.

The authors in [12] are interested to the use of IEEE 802.22, and proposed an algorithm called "Decentralized Q-learning" based on the multi-agent learning theory to deal with the interference problem caused to PUs. They modeled the secondary network using MAS where the different agents are base stations of the IEEE 802.22 WRAN. They proved that the proposed MAS is able to automatically learn the optimal policy to maintain protection for PU against interference.

The authors of [21] and [19] used the MAS to design a new cognition cycle with complex interaction between PU, SU and wireless environments and they used the hidden Markov chains to model the interactions between users and the environment. The results of this approach have shown that the algorithm can guarantee fairness among users.

What could make the use of MAS in the CR interesting and more concrete is the existence of a simulation framework to test the proposed works and approaches. This is precisely what the authors propose in [9]. Their platform allows studying the emerging aspect, the behaviors of heterogeneous CR networks. 


\section{QUALITY OF SERVICE IN Video CONFERENCING}

With the emergence of new services such as video conferencing and video streaming, the need to treat the frames one by one and to know how differentiate services becomes primordial.

An interactive video conferencing cannot tolerate long delays because there is not enough time to retransmit lost packets. Lost packets or very late ones are simply ignored which will cause deterioration of images and sound.

With a network that provides an acceptable throughput, we should control the delay (between the transmission and reception of a packet), jitter (delay variation), usually this type of applications does not tolerate large jitter to not damage the image and sound and of course we must also control the loss of images.

However, we think that $\mathrm{CR}$ nodes possess the necessary qualities to ensure the connection continuity of video conferencing and thus ensure a good quality of service.

In the literature, we found that to have a good QoS in video conferencing, it is necessary that:

- Throughput must be $>384 \mathrm{~Kb} / \mathrm{s}$.

- Delay must be $<200 \mathrm{~ms}$.

- Jitter must be $<30 \mathrm{~ms}$.

- $\quad$ Packet loss must be $<1 \%$.

However, as we don't have real data used in the CR and it is the case of the whole community, we had to play the role of the expert to assign the needed data for our simulation.

\section{Problematic and Proposed solutions using a Single CRMT}

The Figure 1 below shows a path followed by a mobile subscriber when it switches to an area where the signal quality drops to an unacceptable level (shown in red) due to a gap in coverage, we assume that the client uses video conferencing over the route.

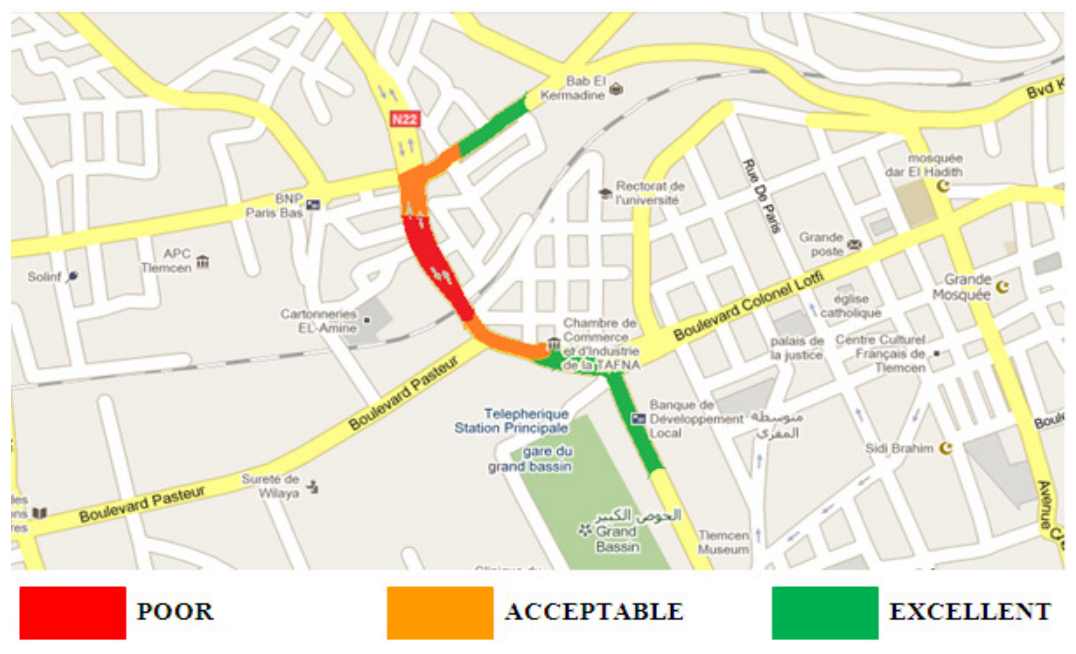

Figure 1 . Signal quality associated to a cognitive radio 


\subsection{Proposed Solution}

After several incidents, the CR should be aware of the problem. Then, through some geolocations or the ability to learn the time of the day when this happens, the radio can anticipate the difference in coverage and know the necessary signal to the base station to change characteristics of the signals when the user approaches the deficient coverage.

\subsection{Application}

As mentioned above, we will use video conferencing in the case of a mobile user who needs to take a path where the signal quality drops to an unacceptable level due to a gap in coverage, giving a very low QoS. This can be remedied by using the CR, but problematic arises: WHEN and WHY to use cognitive radio?

Most research related to the QoS of video conferencing, take into account throughput as pertinent parameter. For this reason, we choose the "Throughput" as a single pertinent parameter for our application. For this, a throughput classification is required, and as we play the role of the expert, we have created our own database following certain rules in order to apply our approach. The database was divided into two parts, the first one for learning and the second one for testing. The value of the throughput will change almost every time it is measured during the day even on the same route, for this reason, our measures have been taken into account for 5 weeks at 3 different intervals of the day ( $8 \mathrm{am}-11 \mathrm{am}, 11 \mathrm{am}-3 \mathrm{pm}, 3 \mathrm{pm}-5 \mathrm{pm}$ ) excluding weekend.

We proposed to affect throughput on three classes:

- Gold for samples where the throughput is greater than $384 \mathrm{~Kb} / \mathrm{s}$, ensuring $100 \%$ quality satisfaction of the user.

- Silver for samples where the throughput is between $160 \mathrm{~Kb} / \mathrm{s}$ and $384 \mathrm{~Kb} / \mathrm{s}$, of acceptable quality.

- Bronze for samples where the throughput is less than $160 \mathrm{~Kb} / \mathrm{s}$. This means that video conferencing is not satisfactory, and it is the class that interests us because this is when we use the $\mathrm{CR}$.

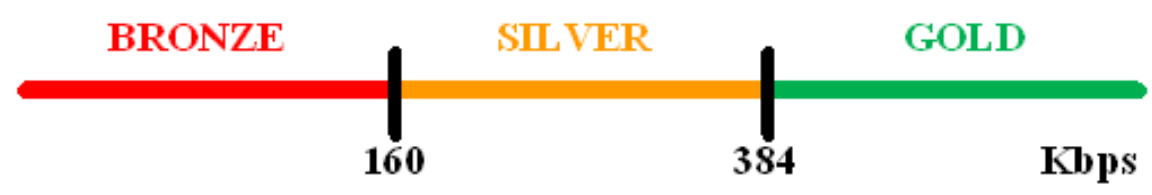

Figure 2. Database classes

\subsubsection{First Question "WHEN"?}

For the data classification, we used three different algorithms derived from the field of machine learning.

- The k-nearest neighbor's algorithm (K-NN) which is a supervised classification algorithm.

- The multilayer perceptron algorithm (MLP of neural networks).

- The C4.5 algorithm of decision trees. 
International Journal of Distributed and Parallel Systems (IJDPS) Vol.3, No.2, March 2012

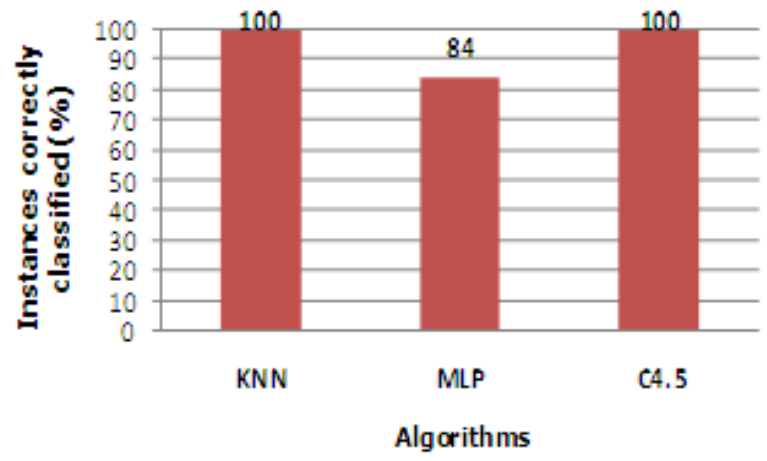

Figure 3. Best scores obtained with the three algorithms

We remark that, the multi layer perceptron has not given satisfactory results compared with the other algorithms; however we did not get $100 \%$, despite the change of parameters such as the number of hidden layers, the number of epochs and the learning rate.

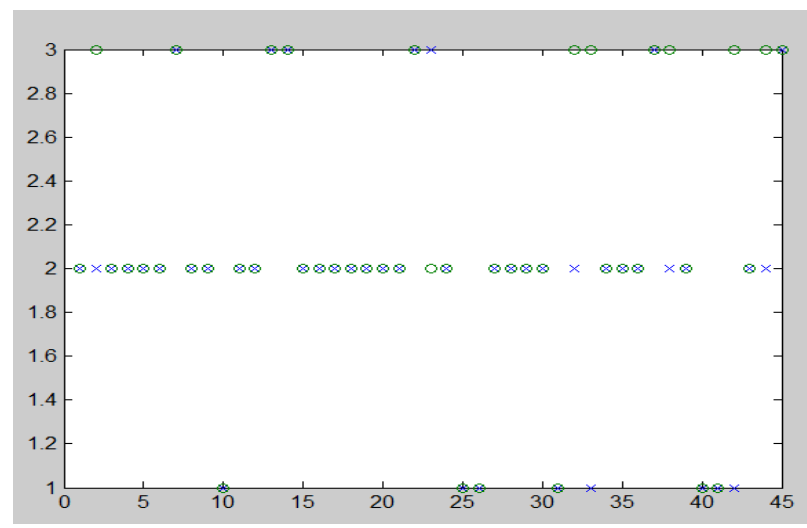

Figure 4. Neural Network Classification

We remark also that the two other algorithms (KNN and C4.5) have given results of $100 \%$, however if we take a look at the generated tree, we see is not exactly what we want because for him the GOLD class starts from a rate of $380 \mathrm{~Kb} / \mathrm{s}$ instead of $384 \mathrm{~Kb} / \mathrm{s}$, this can generate after mistakes with misclassifying some instances.

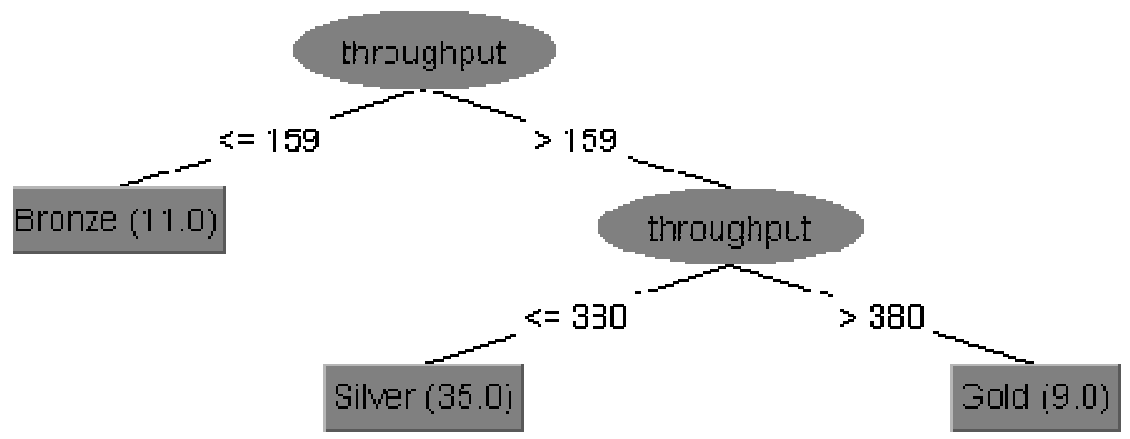

Figure 5. Decision tree 
International Journal of Distributed and Parallel Systems (IJDPS) Vol.3, No.2, March 2012

Concerning the K-NN algorithm, it was tested with several values of $\mathrm{K}$ on the test database and each time the result was different, but it is satisfactory until the value of $\mathrm{K}=6$.

For our approach, we chose this algorithm, as it gave the best results (shown below) in terms of reliability and Clustering.

Table 1 Classification obtained with KNN

\begin{tabular}{|l|l|l|l|l|}
\hline \multicolumn{1}{|c|}{ Values of K } & \multicolumn{1}{c|}{$\begin{array}{c}\text { Instances correctly } \\
\text { classified }\end{array}$} & \multicolumn{2}{c|}{$\begin{array}{c}\text { Misclassified } \\
\text { Instances }\end{array}$} \\
\hline $\mathrm{K}=1$ & 20 & $100 \%$ & 0 & $0 \%$ \\
\hline $\mathrm{K}=2$ & 19 & $95 \%$ & 1 & $5 \%$ \\
\hline $\mathrm{K}=3$ & 19 & $95 \%$ & 1 & $5 \%$ \\
\hline $\mathrm{K}=4$ & 18 & $90 \%$ & 2 & $10 \%$ \\
\hline $\mathrm{K}=5$ & 18 & $90 \%$ & 2 & $10 \%$ \\
\hline $\mathrm{K}=6$ & 18 & $90 \%$ & 2 & $10 \%$ \\
\hline
\end{tabular}

For $\mathrm{K}=1$ : All the throughput examples were well posted in their appropriate class.

Whereas, with $\mathrm{K}=2$, one sample was misclassified, we notice that in the graph.

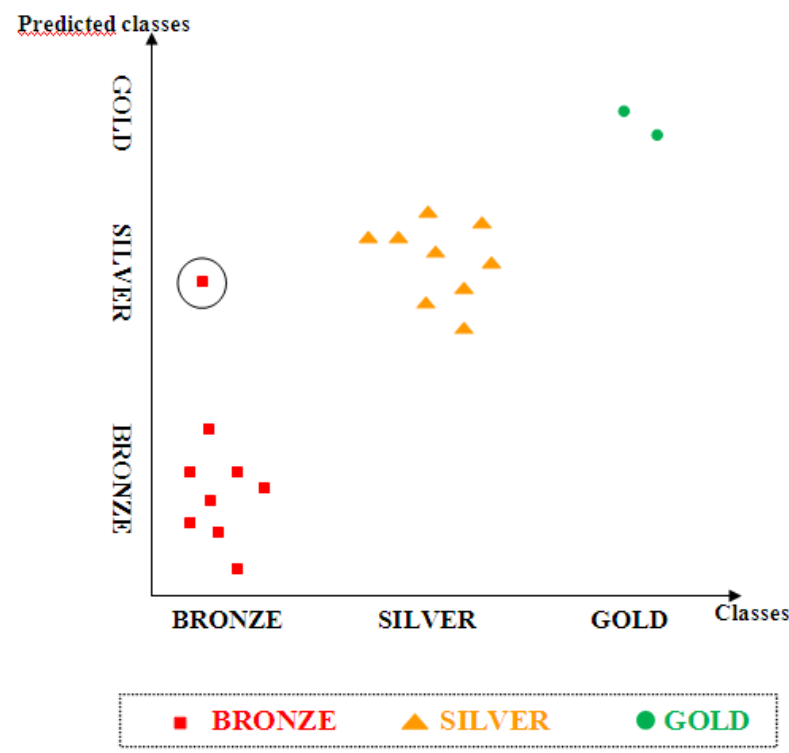

Figure 6. Classification result of $\mathrm{KNN}$ with $\mathrm{K}=2$

Note: items close to the threshold of a class are misclassified. For example, the instance which have a throughput of $159 \mathrm{~Kb} / \mathrm{s}$ was ranked on Silver class instate of the Bronze one, for the other values of $\mathrm{K}$, the result is more divergent.

Instance: 13

Throughput: 159.0

Predicted Class: Silver

Class: Bronze

Figure 7. Instance misclassified 
International Journal of Distributed and Parallel Systems (IJDPS) Vol.3, No.2, March 2012

Report: according to the results of the classification, the cognitive radio will be activated each first day of week from $8 \mathrm{am}$ to $11 \mathrm{am}$ and from $3 \mathrm{pm}$ to $5 \mathrm{pm}$, and every Wednesday from $8 \mathrm{am}$ to $5 \mathrm{pm}$ because in these intervals the throughput belongs to the Bronze class $<160 \mathrm{~Kb} / \mathrm{s}$. So, through this, the question When is answered.

For more general rules, it would be interesting to consider the other QoS video conferencing parameters and classify these data using other methods of artificial intelligence such as fuzzy logic and genetic algorithms.

\subsubsection{Second Question "WHY"?}

Now, We will justify the usefulness of the CR, this by supposing that the spectrum sensing is already done by the receiver of our mobile terminal which is in this case a multimode wireless communication terminal (MWCT), so capable to support multiple access technologies such as GSM, UMTS or WiMAX.

Considering that, the spectrum is not used at $100 \%$, we can represent the frequency bands into two sets: the first contains the occupied bands and the second contains the free ones.

The mobile terminal must switch to an unused frequency band among those available in the free bands set.

For our approach, scenarios were studied to show the usefulness of the CR, this based on the number of free bands and the time of use of each one, we identified three possible scenarios:

- Favourable (best case): the receiver detects a free band and uses it during all the way without any interruption caused by the primary user.

- Unfavourable (worst case): the receiver does not detect any free band (empty set) or it detects some bands but their use interferes with the primary users. In this case the $\mathrm{CR}$ is not used because the secondary user should not disturb the primary users.

- Common ( $\mathrm{N}$ frequency with $\mathrm{N}$ hops): the terminal uses a free band $\mathrm{b} 1$, then there is an interruption caused by the primary user, so it switches to another free band b2 (he made a hop). If the primary user of b2 needs his band, the secondary user must switch again, and so on, until the end of the condition when he opts for the CR (before returning to his initial frequency band), he will have done $\mathrm{N}$ hops.

For our application, we have based on the number of hops done by the CR during all the way of the secondary user. In each of the scenarios mentioned above we calculated the time of interruption which is the required time for the terminal to access a free band and use it.

The time of interruption will be defined as follows:

\section{T.interruption $=($ T.sensing $+T$. establishment $) \times$ number of hops.}

The sensing time is the required time to detect a free band, it is negligible compared to the time of establishment, it is even included in the time of establishment for some algorithms which treat the diagonal handover (switching between wireless networks which use the subjacent technologies such as the standard IEEE 802) or the vertical handover (switching from one access technology to another).

In the literature, we found that the time of establishment necessary to exploit a free frequency band of another technology is 5 seconds on average [5] and [6].

T.sensing $<<$ T.establishment, which gives us: T.interruption $=T$. establishment $\mathbf{x}$ number of hops.

We call time of rupture: the necessary time to return to the initial frequency band. So, it is the sum of the time of interruption and the time of use of each band, knowing that the time of use can differ from a band to another according to the primary user. 
International Journal of Distributed and Parallel Systems (IJDPS) Vol.3, No.2, March 2012

\subsubsection{Experimentation Results}

To support our proposal and to better understand, we compared the QoS with and without cognitive radio (case without CR means that our terminal has only one access technology and works on the same frequency band).

The graphs below illustrate this comparison. For being readable, we supposed that the maximum time of rupture is $5 \mathrm{~min}$ and that the free band set contains 7 bands.

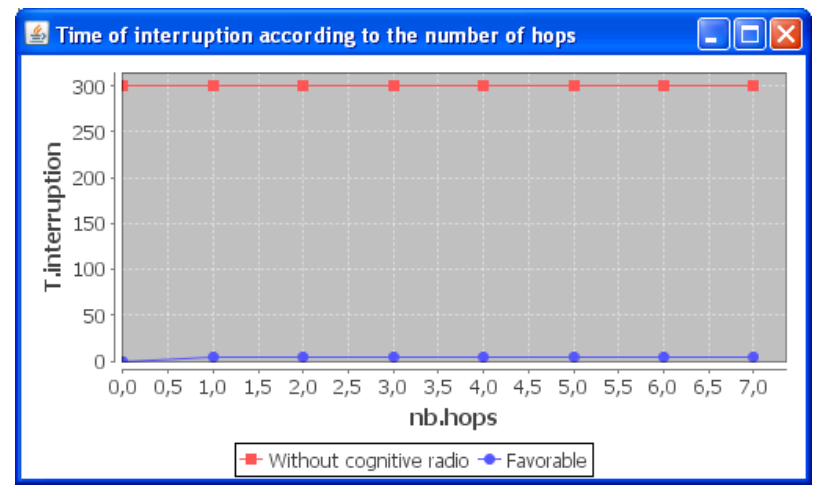

Figure 8. Comparison between the favourable scenario and the case without cognitive radio

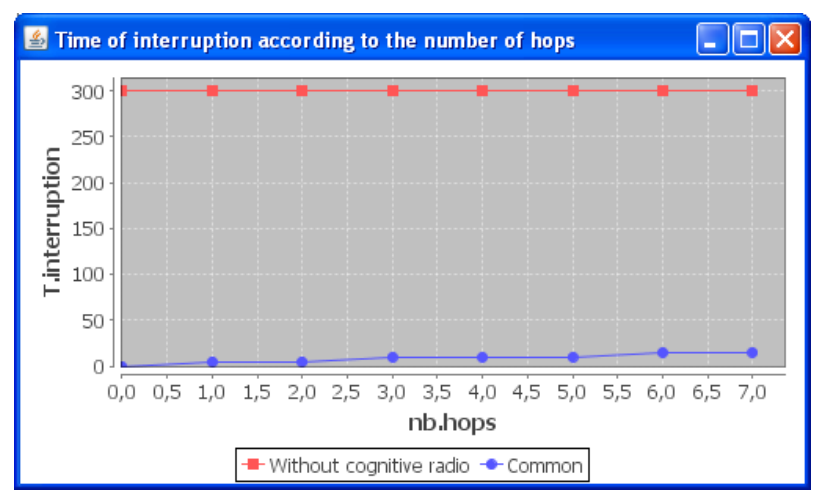

Figure 9. Comparison between the common scenario and the case without cognitive radio

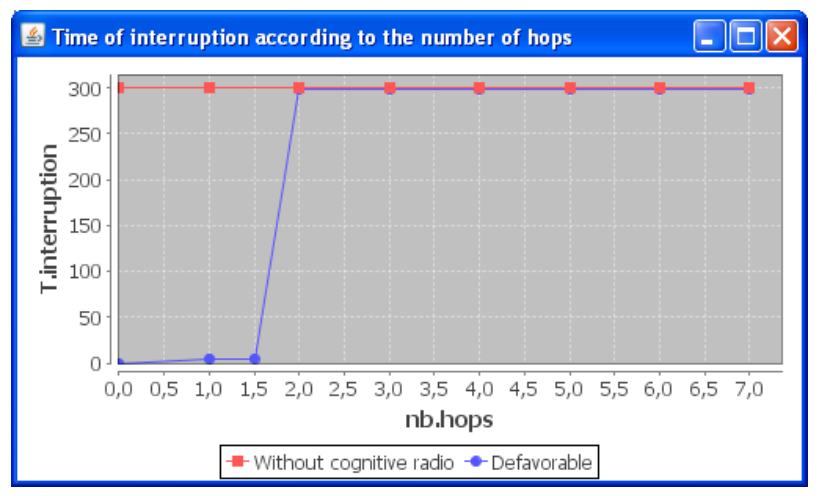

Figure 10.Comparison between the unfavourable scenario and the case without cognitive radio 
International Journal of Distributed and Parallel Systems (IJDPS) Vol.3, No.2, March 2012

\subsubsection{Results Interpretation}

Modeled graphs above represent the downtime depending on the number of hops performed. We note that whatever the number of hops, the case with the CR is much better than that without CR.

According to the first graph, of course we lost 5 seconds to connect to the new band but we see that the CR has saved us 295 s which is the time without interruption.

For the second graph, we performed three hops, which means that the downtime is $5 \mathrm{~s}$ x 3 hops: $15 \mathrm{~s}$. Despite this, the case with CR is always better.

On the third graph, a single hop was made but the connection was interrupted by the primary user at the beginning, so we took advantage of the CR for a few seconds.

Result: whatever the number of hops performed, the use of cognitive radio is always more efficient.

\section{Conclusions}

We presented in this paper a new approach that uses Cognitive Radio to improve wireless communication for a cognitive radio mobile terminal by enhancing the QoS of video conferencing application. Our contribution is positioned in learning from events (machine learning). Our expert role has allowed us to choose the throughput parameter to perform a classification that allows the terminal used to gain experience for future events that means that it will know when and where it will activate the cognitive radio.

The usefulness of cognitive radio is a hypothesis that has been proved based on the required time for a connection to a new frequency band, and this, whatever of the number of frequency bands that a terminal used to remedy a failed connection.

Different approaches using the MAS in the CR are studied, those offering cooperation between SUs only, others offer a cooperation between primary and SUs and those proposing to include a broker agent to negotiate the spectrum, knowing that the most works studied are using reinforcement learning.

In our future work, we think we can improve the wireless links reliability and ensure good quality of service to CR mobile terminals [26] [27] [28] by integrating MAS. We will seek also to reduce the impact of mobility on cognitive radio communications by building predictive models of mobility by referring to previous work such as [29] and [30].

\section{REFERENCES}

[1] J. Mitola, (1999) "Cognitive radio - model-based competence for software radios", Licentiate Thesis, KTH, Stockholm.

[2] J. Neel, (2006) "Analysis and Design of Cognitive Radio Networks and Distributed Radio Resource Management Algorithms", Faculty of the Virginia Polytechnic Institute and State University.

[3] C. Clancy et al, (2007) "Applications of machine learning to cognitive radio networks", IEEE Wireless Communications, vol. 14, no. 4.

[4] E. hossain, D. Niyan, Zhu Han, "Dynamic Spectrum Access and management in cognitive radio networks", Cambridge University Press 2009.

[5] S. Busanelli et al, (2011) "Vertical Handover between WiFi and UMTS Networks: Experimental Performance Analysis", International Journal of Energy, Information and Communications Vol. 2 , Issue 1. 
International Journal of Distributed and Parallel Systems (IJDPS) Vol.3, No.2, March 2012

[6] Z. Daia et al, "Vertical handover criteria and algorithm in IEEE 802.11 and 802.16 hybrid networks", Laboratoire de Motorola Paris.

[7] Atiq Ahmed et al, (2011) "An agent Based Architecture for Cognitive Spectrum Management", Australian Journal of Basic and Applied Sciences, 5(12): 682-689.

[8] Chu Tian et al, (2010) "Spectrum Trading in Cognitive Radio Networks An Agent-based Model under Demand Uncertainty”, Global Telecommunications Conference.

[9] Dzikowski Jacek, Cynthia Hood, (2009) “An Agent-Based Simulation Framework For Cognitive Radio Studies", Simutools '09 Proceedings of the 2nd International Conference on Simulation Tools and Techniques.

[10] Galindo-Serrano Ana, Lorenza Giupponi, (2009) "Aggregated Interference Control for Cognitive Radio Networks Based on Multi-agent Learning”. Proceedings of the 4th International Conference On CROWNCOM.

[11] Gaurav S, Kasbekar, Sarkar S, (2010) "Spectrum auction framework for access allocation in cognitive radio networks”, IEEE.ACM Transactions on Networking, vol. 18, pp. 1841 - 1854.

[12] Jiandong Li, Chungang Yang, (2010) "A Markovian Game-Theoretical Power Control Approach In Cognitive Radio Networks: A Multi-Agent Learning Perspective”, Wireless Communications and Signal Processing (WCSP).

[13] Letaief Ben, K., Wei Zhang, (2009) "Cooperative Communications for Cognitive Radio Networks", Proceedings of the IEEE.

[14] Li H, (2009) "Multi-agent Q-Learning of Channel Selection in Multi-user Cognitive Radio Systems: A Two by Two Case”, Systems, Man and Cybernetics.

[15] Lim Kok et al, (2011) "Achieving Context Awareness and Intelligence in Distributed Cognitive Radio Networks: A Payoff Propagation Approach", Workshops of International Conference on Advanced Information Networking and Applications.

[16] Mir Usama, Leila Merghem-Boulahia, Dominique Gaïti, (2010) “A Cooperative Multiagent Based Spectrum Sharing", Sixth Advanced International Conference on Telecommunications.

[17] Mir Usama, Leila Merghem-Boulahia, Dominique Gaïti, (2010) "Dynamic Spectrum Sharing in Cognitive Radio Networks: a Solution based on Multiagent Systems". International Journal on Advances in Telecommunications, vol 3 no 3 \& 4 .

[18] Usama Mir, LeilaMerghem-Boulahia, Dominique Gaïti. (2010). COMAS: A CooperativeMultiagent Architecture for Spectrum Sharing. EURASIP Journal onWireless Communications and Networking, Volume 2010, Article ID 987691.

[19] Mir Usama, Leila Merghem-Boulahia, Dominique Gaïti, (2009) "Utilization of a Cooperative Multiagent System in the Context of Cognitive Radio Networks". (C) Springer-Verlag Berlin Heidelberg 2009, LNCS 5844, pp. 100-104.

[20] Mir Usama, Leila Merghem-Boulahia, Dominique Gaït, (2010) "Multiagent Based Spectrum Sharing Using Petri Nets". Y. Demazeau et al. (Eds.): Trends in PAAMS, AISC 71, pp. 537546. springerlink.com (C) Springer-Verlag Berlin Heidelberg.

[21] Qi Zhao, Qin shi, Wu Zhijie, (2011) "Self-Organize Network Architecture for Multi-Agent Cognitive Radio Systems", International Conference on Cyber-Enabled Distributed Computing and Knowledge Discovery.

[22] Raiyn J, "Toward cognitive radio handover management based on social agent technology for spectrum efficiency performance improvement of cellular systems Personal", IEEE 19th International Symposium.

[23] Trigui Emna, Moez Esseghir, Leila Merghem Boulahia, (2011) "Gestion dynamique du spectre entre terminaux radio cognitive mobiles”, CFIP 2011 - Colloque Francophone sur 1 Ingénierie des Protocoles. 
International Journal of Distributed and Parallel Systems (IJDPS) Vol.3, No.2, March 2012

[24] Wu Cheng et al, (2010) "Spectrum management of cognitive radio using multi-agent reinforcement learning", AAMAS '10 Proceedings of the 9th International Conference on Autonomous Agents and Multiagent Systems.

[25] Xie Jiang, Howitt Ivan, Raja Anita, (2007) “Cognitive Radio Resource Management Using Multi Agent Systems”, Consumer Communications and Networking Conference.

[26] A. Amraoui, W. Baghli and B. Benmammar, (2012) "Improving video conferencing application quality for a mobile terminal through cognitive radio", 14th IEEE International Conference on Communication Technology (ICCT 2012). Chengdu, China, November 9th-11th. to appear.

[27] B. Benmammar, A. Amraoui and W. Baghli, (2012) "Performance improvement of wireless link reliability in the context of cognitive radio". IJCSNS International Journal of Computer Science and Network Security, Vol.12, No.1, Pages: 15-22.

[28] A. Amraoui and al, (2012) "Toward cognitive radio resource management based on multi-agent systems for improvement of real-time application performance", 5th IFIP International Conference on New Technologies, Mobility and Security (NTMS 2012). Istanbul, Turkey, May 7th-10th. to appear.

[29] N. Samaan, B. Benmammar, F. Krief and A. Karmouch. "Prediction-based Advanced Resource Reservation in a Mobile Environment". 18th IEEE Annual Canadian Conference on Electrical and Computer Engineering, CCECE05, May 1-4, 2005, Saskatoon Inn, Saskatoon, Saskatchewan Canada.

[30] B. Benmammar and F. Krief. "MQoS NSLP: a mobility profile management based approach for advance resource reservation in a mobile environment". Proceedings of the 7th IFIP IEEE International Conference on Mobile and Wireless Communications Networks (MWCN 2005). Marrakech, Morocco. September 19-21, 2005. 
Authors :

Asma Amraoui, born in 1989. Graduate of BS in Computer Science in 2009 from the UABT (Université Abou Bekr Belkaïd Tlemcen) in Algeria. Obtained the Master degree in Computer Science option "Intelligent Models and Decisions" in 2011.

Currently she is a $\mathrm{PhD}$ candidate; she is preparing a doctoral thesis on a topic of research that explores the use of Artificial Intelligence techniques in the cognitive radio networks.

She is attached to the LTT laboratory (Laboratory of Telecommunications of Tlemcen) in Algeria.

Badr Benmammar received the B.Sc. in Computer Engineering, with high honors, from the USTO (Université des Sciences et de la Technologie Oran), Algeria, in 1999. He received the M.Sc. in Computer Science from Paris 13 University, France, in 2002. He received the Ph.D degrees in Computer Science from the Bordeaux 1 University, France, in 2006. Badr Benmammar was associate professor from 2010 at UABT (Université Abou Bekr Belkaïd Tlemcen), Algeria and research fellow at CNRS LaBRI Laboratory of the University of Bordeaux 1 until 2007. He is performing his research activities in Laboratory of Telecommunication Tlemcen, UABT, Algeria. His main research activities concern cognitive radio network, quality of service on mobile and wireless networks, end-to-end signaling protocols and Agent technology. His work on quality of service has led to many publications in journals and conferences (Annals of telecommunications, International Journal of Network Management, International Journal of Computer Science and Network Security, WiMob, NetCon, ICT, MWCN, NTMS ...).

Francine Krief is Deputy Director General in charge of Research at IPB (Institut Polytechnique de Bordeaux). She is a Professor at CNRS LaBRI Laboratory, UMR 5800. Francine Krief obtained the HDR degree (Habilitation à Diriger des Recherches) at University of Paris 6 on context-aware management, in December 2003. Her main research activities concern self-management for wired and wireless networks, end-to-end signalling protocols and wireless sensors networks. Her work on network management has led to many publications in journals and conferences.

Fethi Tarik Bendimerad was born in Sidi Bel Abbès, Algeria, in 1959. He received the Ingénieur d'Etat diploma in 1983 from Université des Sciences et Technologies d'Oran, Algeria, and the Doctorate Degree from Université de Nice Sophia Antiplois, France, in 1989, respectively.

$\mathrm{He}$ is a Senior Lecturer within Tlemcen University and the Director of the Telecommunications Laboratory. His field of interest is microwaves and radiation.
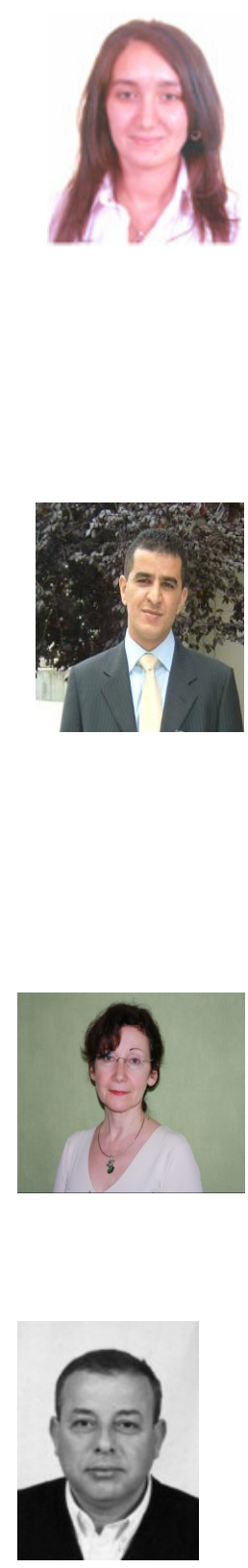